\title{
Clinical research in an academic psychiatry department: some general principles and case studies
}

Australasian Psychiatry 2017, Vol 25(6) 596-599 (C) The Royal Australian and New Zealand College of Psychiatrists 2017 Reprints and permissions: sagepub.co.uk/journalsPermissions.nav DOI: 10.1177/1039856217734675 journals.sagepub.com/home/apy

@SAGE

\begin{abstract}
Philip Keightley Clinical Lecturer in Adult Psychiatry, Academic Unit of Psychiatry and Addiction Medicine, Australian National University Medical School, Canberra, ACT, and; Staff Specialist, ACT Health, Canberra, ACT, Australia
\end{abstract}

Paul Maguire Lecturer in Adult Psychiatry and Acting Co-Deputy Head, Academic Unit of Psychiatry and Addiction Medicine, Australian National University Medical School, Canberra, ACT, and; Staff Specialist, ACT Health, Canberra, ACT, Australia

Rebecca E Reay Lecturer and Senior Research Coordinator, Academic Unit of Psychiatry and Addiction Medicine, Australian National University Medical School, Canberra, ACT, and; ACT Health, Canberra, ACT, Australia

Jeffrey C Cubis Senior Lecturer in Child and Adolescent Psychiatry, Academic Unit of Psychiatry and Addiction Medicine, Australian National University Medical School, Canberra, ACT, and; Senior Staff Specialist, ACT Health, Canberra, ACT, Australia

Peggy Craigie Student Coordinator and Departmental Administrator, Academic Unit of Psychiatry and Addiction Medicine, Australian National University Medical School, Canberra, ACT, and; ACT Health, Canberra, ACT, Australia

Jeffrey CL Looi Associate Professor Psychiatry, Psychiatry of Old Age \& Neuropsychiatry, \& Acting Discipline Lead \& Head, Academic Unit of Psychiatry and Addiction Medicine, Australian National University Medical School, Canberra, ACT, and; Senior Staff Specialist, ACT Health, Canberra, ACT, Australia

\begin{abstract}
Objective: This paper gives guidance for developing collaborative clinical research within an academic psychiatry department.

Methods: We describe the experience at the Australian National University Medical School, and present three case studies.

Results: The results reveal that general principles include, but are not limited to, intellectual curiosity, mentorship, collaboration and protected time.

Conclusions: We conclude that a particular strength of a new research department at a medical school may be close collaborative research within clinical settings.
\end{abstract}

Keywords: research, academic psychiatry, psychiatry, medical school

$\mathrm{A}$ part from developing staffing, a curriculum and clinical placements, the next important task for an academic psychiatry department is to conduct and support clinical research. Much has been written about the decline of academic psychiatry, as well as strategies for its revitalisation, often with little detail on how to bridge the gap to practical implementation. We begin by describing some general approaches to clinical research and linking to specific case studies of academic psychiatrists. We have placed a particular focus on opinions derived from our own experiences. Others have written accounts of the challenges facing Australasian academic psychiatry and have given practical advice regarding the scholarly project. ${ }^{1,2}$

\section{Beginning research in a new academic unit}

The time for planning and conduct of research will be limited by the core tasks of curriculum development, teaching and examinations. This often means that initial research plans, while remaining innovative, may need to

\section{Corresponding author:}

Philip Keightley, Academic Unit of Psychiatry and Addiction Medicine, Australian National University Medical School, Building 4, Level 2, The Canberra Hospital, PO Box 11, Woden, ACT 2605, Australia.

Email: philip.keightley@act.gov.au 
Table 1. Clinical research in a new academic unit

$\begin{array}{ll}\text { Why research - clinical } & \text { - Clinical epidemiologic questions: e.g. how many people over } 65 \text { have mental health problems } \\ \text { questions } & \text { - Clinical questions: e.g. how do the health beliefs of persons with schizophrenia influence the } \\ & \text { - Clinical service questions: e.g. what are the outcomes of crisis service provision? } \\ \text { What methods to research } & \text { - Therapeutic questions: what is the most effective psychotherapy for perinatal depression? } \\ & \text { - Qupulation surveys for epidemiology - access existing or collect new databases } \\ & \text { - Case-control studies for rarer disorders } \\ \text { - Clinical trials for interventions } & \text { - Single researcher } \\ \text { - Collaboration - within department and/or with external researchers } & \text { - Collaboration - undergraduate and postgraduate researchers } \\ & \text { - Enrolling in a postgraduate research degree } \\ & \text { - Seeking supervisors and mentors }\end{array}$

\section{Table 2. Getting started on clinical research}

1. Frame a research question

2. Decide on methodology

3. Learn required research skills

4. Seek collaborators with requisite skills

5. Frame pilot study
a. Seek ethics approval
b. Seek funding
c. Conduct pilot
d. Analyse pilot
e. Evaluate pilot

6. Redesign substantive study based on feedback of 5

7. Substantive study
a. Seek ethics approval
b. Seek funding
c. Conduct
d. Analyse
e. Evaluate

8. Publish

9. Plan new research based on above

collaboratively synergise with the existing clinical and research environment within the associated health service, medical school and broader university environment. Such considerations are outlined in Table 1.

\section{Practicalities}

The general steps of developing research will still apply whatever the setting (see Table 2), and ethics approval often remains the rate-limiting step where protocols, funding, individuals approved to be involved, questionnaires, plain language statements and divisional approval all have to be set in concrete. In clinical settings, this requires a thorough process of consultation to ensure that studies are well designed, feasible and address meaningful issues for the service.

\section{Elements of a framework for developing research capacity}

To facilitate the development of clinical research, it is necessary that the senior staff of an academic unit be active in clinical research. Given the very limited time that early career academic researchers will have in which to conduct their research, they cannot afford to start off course. Similarly, as early career academic researchers begin to plot their research plans, they will need advice and encouragement from seasoned navigators, including examples of worked research proposals, ethics applications and grant applications. Experienced researchers can also facilitate access to key advisors, collaborators and resources internal and external to the medical school. Finally, senior academic staff can help early career researchers strategically plan their research in the context of the broader field and, more specifically, to advance their careers as effective researchers.

\section{Case studies}

The following case studies describe the experiences of two early career clinical psychiatrist researchers within the Academic Unit of Psychiatry and Addiction Medicine, Australian National University (ANU) Medical School, Paul Maguire and Philip Keightley, who both conducted clinically relevant research within their own practice settings. Rebecca Reay and Jeff Cubis then describe a 
highly embedded trauma-focused care research project, where an initial investigation within a child, adolescent and perinatal mental health service paired with an immediate team-level intervention led to measurable benefits.

\section{Paul Maguire}

Compelled by a strong interest in, and commitment to, the physical health of people with a mental illness, I launched a research project exploring how people with schizophrenia perceive the risks associated with pandemic and seasonal influenza, and what protective behaviours they may be willing to take. The initiation of this project coincided with the emergence of the H1N1 swine influenza pandemic in April of 2009, which gave it added impetus as there were many medical clinicians actively engaged in attempting to mitigate harmful outcomes related to this outbreak.

Two of my three PhD-assigned panel supervisors were members of the Academic Unit of Psychiatry and Addiction Medicine at the ANU (including Professor Beverley Raphael, as principal supervisor), which facilitated meetings and discussions about my research project. In addition to regular formal supervision sessions, there were monthly "group $\mathrm{PhD}$ " meetings, moderated by Professor Raphael. On these occasions, five to six PhD candidates interacted with each other, presenting their own research projects, ideas and challenges, as well as providing constructive feedback on other candidates' issues and research conundrums. I found these sessions extremely helpful, as a setting for stimulating and productive "cross-pollination" of ideas.

My $\mathrm{PhD}$ research project generated publications ${ }^{3}$ and the unit provided a rich resource in terms of co-authors and generous editing advice from its members. In addition, following submission of abstracts arising from the research, I was selected to deliver several oral presentations both within Australia and overseas. The Academic Unit of Psychiatry and Addiction Medicine provided an environment where I was able to carry out "practice runs" and incorporate the candid, thoughtful and very helpful suggestions and reflections given in feedback by my colleagues.

In addition to ideas and knowledge, the unit fostered a psychologically positive milieu of support, inspiration, encouragement and curiosity. I felt privileged to be part of a cohesive, vibrant and closely knit team of people, most of whom were both clinicians and researchers. There has always been a strong collegial spirit, positive attitudes, good humour, unfailing sense of enquiry and desire to extend the current state of knowledge and understanding of mental illnesses and the challenges faced by those suffering from them.

\section{Philip Keightley}

In 2014-2015 I investigated the psychological predictors of fatigue in a local group of inflammatory bowel disease
(IBD) patients, focusing in particular on attachment styles. I am currently preparing for publication. This work emerged from a general interest in the psychological aspects of bowel disease, ${ }^{4}$ and from a clinic I established as a senior registrar in the IBD clinic at Canberra Hospital, where I provided brief psychotherapy interventions. I became curious about attachment style as a predictor of fatigue in this population. I collected survey data on fatigue, attachment style and other psychometric variables, and clinician and laboratory ratings of disease severity.

It would not have been possible to devise and carry out the research plan without key ingredients. I needed the practical support of the Hospital-in-the-Home service and Professor Paul Pavli, who, because I was embedded within the IBD service, were generously willing to help with data collection. I also needed dedicated time away from clinical responsibilities, and I am reminded of a recent interview in which Sir David Goldberg told budding psychiatric researchers to "arrange your personal timetable so that whole days are devoted to writing up your research". ${ }^{5}$ For "whole days", I am grateful to ACT Health, who provided a part-time Fellow position paying a registrar salary, based at the ANU Medical School. This salaried teaching and research position continues for my registrar successors, and has been maintained in the face of the well-known clinical staffing pressures experienced in acute mental health services. The continuing existence of this position is a testament to Professor Raphael's resolute but diplomatic style.

Odysseus placed his son Telemachus into the care of his trusted friend Mentor, who became a father and guide. ${ }^{6}$ There are many low points in a research project. Recruitment can be arduous, administrative structures obstructive, family and life stressors unavoidably distracting and clinical work urgent. Encouragement and unconditional, genuine interest in my endeavour from my supervisors gave me the energy to keep pushing. Other researchers, I am sure, will be able to relate to the motivating force of the thought "what would my supervisor think about this?".

\section{Rebecca Reay and Jeff Cubis}

Trauma-informed care is emerging as a fundamental issue for contemporary mental health services. Consultations with clinical staff and senior managers revealed recognition of the impact of trauma in the lives of clients. However, there was less consensus about the size of the problem and how to sensitively screen, respond to and manage clients with complex trauma histories. Research into the area was also seen as an important team-building activity and a stimulus for wider research interest in the service. Together with representatives from the Perinatal, Child and Adolescent Mental Health Service, we initiated a multifaceted pilot project to address this gap in current service delivery. 
Table 3. General principles to facilitate early career research

- Maintain intellectual curiosity in clinical settings

- Approach suitable mentors and supervisors

- Actively reach out to research collaborators

- Seek appropriate advice and support from colleagues within and outside department

- Learn new research skills including, but not limited to, methodology, data analysis and writing for publication

- Seek protected time to think, plan and write

- Consider enrolling in a postgraduate research degree

To begin, we established a steering committee to oversee the design, implementation and evaluation of the study. A literature review was conducted and we held extensive discussions with clinicians and managers in order to explore their issues and needs. A project was collaboratively developed and approved by the local health ethics committee with seed funding from a local hospital research grant. The project aims were to: 1 ) estimate the prevalence of trauma in the perinatal and child and adolescent mental health services (CAMHS); and 2) increase clinician knowledge, awareness of and confidence in responding to trauma. Critical to the success of the prevalence study was embedding it within the teams: clinicians routinely assessed clients for adversity and trauma experiences and were involved in recruitment and data collection. The findings revealed that $69 \%$ of children and adolescents and $80 \%$ of mothers (in the perinatal period) who accessed the service had experienced a potentially traumatic event. ${ }^{7}$

Our consultations with clinicians about the lack of undergraduate training in trauma-informed or specific interventions, led to the next research phase. In phase 2, we were able to show that trauma-informed care training was associated with improvements in the clinicians' attitudes, knowledge and confidence towards working with clients with trauma histories (manuscript submitted for publication). The next thrust of our research is on the health outcomes for CAMHS clients who receive a trauma-specific intervention. It has been a very rewarding experience to collaborate on research around meaningful service delivery issues and have a positive impact on clinical practice.

\section{Case study comments}

These examples of embedded clinical research were conducted by a new academic psychiatry unit closely linked with a health service. The projects required minimal funding. The local collaborative approach had the particular benefit of strengthening clinical and academic links between clinical services, with flow-on clinical benefits for patients, as well as opening the door for future research collaborations. We have summarised some general principles in Table 3.

\section{Conclusions}

Through strategic planning, dedicated effective work and mentoring, an academic psychiatry unit can build meaningful research links. Such active research participation can iteratively inform teaching and thus improve the quality of clinical medical school education. A medical-school-based academic department may be particularly well placed to bridge clinical practice and university research, potentially leading to innovation in psychiatry. Based on a foundation of clinical research, chance will not only favour being prepared in mind as a clinician, but will favour students similarly through researchinformed teaching.

\section{Acknowledgements}

We dedicate this work to the foundational role of Professor Beverley Raphael, Discipline Lead of the Academic Unit from 2006 to 2016. Thanks to all staff, past and present.

\section{Disclosure}

The authors report no conflict of interest. The authors alone are responsible for the content and writing of the paper. No specific funding was received for this paper.

\section{Funding}

The authors received no financial support for the research, authorship, and/or publication of this article.

\section{References}

1. Henderson S, Porter RJ, Basset D, et al. Why academic psychiatry is endangered. Aust NZJ Psychiatry 2015; 49: 9-12

2. You D, Glozier N and Kealy-Bateman W. The Scholarly Project in the Royal Australian and New Zealand College of Psychiatrists training program: getting up and running in Stage 1. Australas Psychiatry 2017; 25: 185-186.

3. Maguire PA, Reay RE and Raphael B. Correlates of a single-item Self-Rated Mental Health Question in people with schizophrenia. Australas Psychiatry 2016; 24 : 473-477.

4. Keightley PC, Pavli P, Platten J, et al. Gut feelings 2. Mind, mood and gut in inflammatory bowel disease: approaches to psychiatric care. Australas Psychiatry 2015; 23 : 407-410.

5. Goldberg D. An erudite encounter with: Sir David Goldberg. Aust NZ J Psychiatry 2017; 51: 103-104.

6. Homer. Odyssey. London: Penguin Classics, 2003.

7. Reay R, Raphael B, Aplin V, et al. Trauma and adversity in the lives of children and adolescents attending a Mental Health Service. Children Australia 2015; 40: 167-179. 